\title{
Health-related quality of life correlated with the clinical severity of intermittent exotropia in children
}

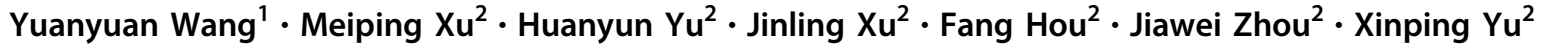

Received: 26 February 2019 / Revised: 16 June 2019 / Accepted: 1 July 2019 / Published online: 12 August 2019

(c) The Author(s), under exclusive licence to The Royal College of Ophthalmologists 2019

\begin{abstract}
Object To evaluate whether the results of the health-related quality of life (HRQOL) is associated with the various clinical aspects of intermittent exotropia in children.

Methods The HRQOL of children and their parents was evaluated prospectively using the Intermittent Exotropia Questionnaire (IXTQ). The deviation angle, stereo function, sensory fusion, and strabismus control were measured.

Results Two hundred and sixty six children with intermittent exotropia (aged 5-17 years) were included in the study. Child HRQOL was significantly correlated with clinical severity; lower IXTQ scores were associated with a larger deviation $(p<0.001$ both for distance and near) and poorer Newcastle control scores $(p<0.001)$. Proxy child HRQOL was significantly correlated with the deviation $(p<0.001)$, Newcastle control scores $(p<0.001)$ and stereo function $(p<0.05)$. Parent HRQOL was associated with their child's deviation $(p<0.01)$ and stereo function $(p<0.005)$. Multiple linear regression analysis suggested that the deviation angle at distance and the Newcastle control score at home were associated with the child's HRQOL.

Conclusion Both the child and their parents' HRQOL showed a trend toward correlating with clinical severity. Large deviation, poor control, and poor stereo function were significantly associated with lower IXTQ scores. The deviation angle at distance and exotropia control at home were associated with the child's HRQOL.
\end{abstract}

\section{Introduction}

Intermittent exotropia is a common form of strabismus [1] that affects $3.3-3.9 \%$ of Chinese children $[2,3]$ and is one of the most common forms of childhood strabismus in Asia [4]. Patients may maintained with normal ocular alignment and binocular sensory function, and the eyes would present a divergent deviation accompanied with the loss of stereoacuity when tired, inattentive or fixing at distance [5]. Patients with intermittent exotropia normally have variable control and various deviation angles; it can also occur with minimal deviation or sustained exotropia for a short period [6-8]. In clinical practice, the angle of deviation,

Xinping $\mathrm{Yu}$

yu-xinping@163.com

1 Department of Ophthalmology, Huzhou Central Hospital, Huzhou, Zhejiang, China

2 The Eye Hospital of Wenzhou Medical University, Wenzhou, Zhejiang, China stereoacuity, and control of the deviation angle or deviation frequency have been commonly used to evaluate the severity and progression of intermittent exotropia in children [6]. Intermittent exotropia also causes a child's parents to worry about their visual perception and makes them sensitive to others' comments about their child's eyes, which affect the health-related quality of life (HRQOL) of children as well as their parents $[9,10]$.

Even the questionnaires for quantifying HRQOL in childhood intermittent exotropia, e.g., "shutting one eye when sunny, waiting for their eyes to clear up, and worrying about their eyes", were designed to be clinically relevant $[11,12]$. However, few studies have evaluated the correlation between the severity of intermittent exotropia and HRQOL. In one recent study by Lim et al. [13], child HRQOL did not correlate with clinical severity. However, given the small sample size (i.e., 63) and relatively narrow spectrum of severity of intermittent exotropia in their study, whether HRQOL is correlated with the clinical severity of this condition remains uncertain. Moreover, whether the HRQOL of children and their parents could be predicted based on the clinical evaluation of the intermittent exotropia remains unknown. For 
this reason, we conducted this prospective study to recruit a large number of child intermittent exotropia patients. In this study, we aim to assess the relationship between HRQOL and the severity of intermittent exotropia.

In particular, we used the Intermittent Exotropia Questionnaire (IXTQ), a condition-specific questionnaire on HRQOL for children with intermittent exotropia and their parents [14], to determine whether there is a correlation between HRQOL and the severity of intermittent exotropia (i.e., angle of deviation, control and binocular vision) in children with intermittent exotropia. Factors correlating with patients' HRQOL were also evaluated.

\section{Materials and methods}

\section{Patients and methods}

This prospective study recruited children with intermittent exotropia at the Eye Hospital of Wenzhou Medical University. The inclusion criteria were as follows: (1) 5 to 17 years old when enrolled in the study; (2) intermittent exotropia measured through the prism and alternate cover test (PACT), which was not less than ten prism diopters (PD) at near and at distance; (3) no "A", "V" pattern or vertical deviation; (4) best corrected visual acuity better than 20/30 in each eye; (5) less than two lines of visual acuity difference. Patients were excluded from the study if they had a history of ocular surgery or strabismus surgery, vision training or patching treatment, sensory or paralytic exotropia, nystagmus or other conditions limiting ocular movement or associated with facial deformities, neurologic disorders, or any significant medical problems. This study was approved by the Eye Hospital of Wenzhou Medical University Ethics Committee and adhered to the tenets of the Declaration of Helsinki. All participants and their parents provided their written or verbal informed consent to participate in this study, and the ethics committees approved this consent procedure.

Sample size: Lim et al. [13] did not find significant correlation between child HRQOL and clinical severity with 63 patients. We estimated the sample size for our study before recruiting patients. This method assumes the threshold of rejecting the null hypothesis (no correlation) $=$ 0.05 and the probability of failing to reject the null hypothesis under the alternative hypothesis $=0.1$, we expect to observe a correlation with the correlation coefficient of at least 0.2. According to previous described methods [15], the minimum number of patients should be computed as:

$$
N=\left(\frac{Z_{\alpha}+Z_{\beta}}{C_{r}}\right)^{2}+3
$$

where $Z_{\alpha}$ and $Z_{\beta}$ are the standard normal deviate of $\alpha$ and $\beta$, and $C_{r}=0.5 \ln \left(\frac{1+r}{1-r}\right)$ Substitute $\alpha, \beta$ and $r$ with $0.05,0.1$ and 0.2 , respectively, we have $N=259$.

So, we decided to recruit 260 patients and in the end of the study, the actual number of subjects was 266 , recruited between July 1, 2016 and August 31, 2017.

\section{Assessment of the severity of intermittent exotropia}

The severity of intermittent exotropia in our patients was assessed by the size of deviation, binocular function (including sensory fusion and stereoacuity), and the control of the deviation [6]. Ocular deviation was measured using PACT at $5 \mathrm{~m}$ (for distance) and $40 \mathrm{~cm}$ (for near). Sensory fusion was tested using the Worth 4 dot test at $40 \mathrm{~cm}$ (near) and $5 \mathrm{~m}$ (distance) and was classified as normal (without suppression or diplopia) or abnormal (suppression or diplopia). Stereoacuity was tested using TNO stereopsis tests at near $(40 \mathrm{~cm})$ and at distance using an Optec 3500 (Stereo optical, CO., INC., Chicago, IL). Stereoacuity at near and distance was classified as normal (<100 s arc), subnormal stereo $(120-800$ $\mathrm{s}$ arc) or none ( $>800 \mathrm{~s}$ arc or without) [16]. The control of deviation was quantified by the Newcastle control score which has been shown to correlate with disease severity $[17,18]$. In particular, Newcastle control scores for home $(0=$ never, $1 \leq 50 \%$ distance, $2 \geq 50 \%$ distance, $3 \geq 50 \%$ distance and near) and for office at distance and near $(0=$ immediate realignment, $1=$ realign with blink or fixation, $2=$ remain manifest or manifest spontaneously) were assessed [17]. The Newcastle control scores of all patients were examined at near $(40 \mathrm{~cm})$ and distance $(5 \mathrm{~m})$ by the same investigator (WYY) during the study. The sum of the Newcastle scores for home and office [17, 18] was used as the quality of deviation control for further analysis.

\section{Assessment of HRQOL}

All children and their parents were asked to complete the IXTQ $[10,14,19]$. The typical IXTQ includes the following sections: one for children only (i.e., the Child scale), another for the parents about the child (i.e., the proxy scale) and the last for the parents about themselves (i.e., the Parent scale). For each question, a five-point Likert-type scale is used for the following individual responses: never (score 100), almost never (75), sometimes (50), often (25), and almost always (0). However, for the child IXTQ (5-7 years), only three levels are used as follows: not at all (100), sometimes (50), and a lot (0). The final IXTQ score for each participant is calculated as the mean of all items, which ranges from 0 to 100 (worst to best HRQOL) [14, 19].

During our assessment of HRQOL, the children and their parents finished the test separately without any 
communication (verbal or nonverbal) between them. Questionnaires were self-administered with written instructions and were supervised by the same investigator (WYY). If the child or his/her parents had some problems in understanding the questionnaire, a verbal interview was conducted without any explanation or elaboration [14, 19].

\section{Statistical analysis}

The results of patients with different genders or different sensory fusion levels were compared using the two-tailed independent samples $t$-test. Patients with differences in stereo function were compared using one-way analysis of variance. Correlations between IXTQ scores and the angle of deviation or Newcastle control scores were evaluated with the Pearson's correlation. The relationship between strabismus characteristics and demographics with IXTQ scores was assessed with a generalized linear model. All statistics were analyzed using the SPSS 19.0 software package (SPSS Inc., Chicago, IL, USA). $P$-values $<0.05$ were considered significant.

\section{Results}

\section{Summarized results}

Table 1 summarizes the results and clinical details of the 266 patients included in the study. There was no significant correlation between patients' age and their deviations at distance $(r=0.09, p=0.15)$, at near $(r=0.03, p=0.68)$ or Newcastle control scores $(r=0.47, p=0.47)$. The deviations at distance $(t=0.80, p=0.43)$ or near $(t=0.22, p=$ $0.83)$ were also not significantly different between genders. The averaged IXTQ scores were $66.0 \pm 17.8(0-100)$ for the child scale, $68.9 \pm 17.6(2.1-100)$ for the proxy scale and $43.4 \pm 21.9(0-96.7)$ for the parent scale.

\section{Correlation between HRQOL and the severity of intermittent exotropia}

Figure 1 shows the relationships between the Child IXTQ scores and deviations at distance (Fig. 1a) or near (Fig. 1b).
Table 1 Summarized results of patients

\begin{tabular}{|c|c|c|c|}
\hline & & & Number \\
\hline \multicolumn{4}{|l|}{ Demographics } \\
\hline Age (years) & & & $5-17,(9.9 \pm 3.0)$ \\
\hline \multirow[t]{2}{*}{ Gender } & \multicolumn{2}{|l|}{ Boy } & $144(54 \%)$ \\
\hline & \multicolumn{2}{|l|}{ Girl } & $122(46 \%)$ \\
\hline \multirow[t]{2}{*}{ Family history of strabismus } & \multicolumn{2}{|l|}{ Yes } & $42(15.8 \%)$ \\
\hline & \multicolumn{2}{|l|}{ No } & $224(84.2 \%)$ \\
\hline \multirow[t]{3}{*}{ Accompanying parent } & \multicolumn{2}{|l|}{ Mothers } & $114(43 \%)$ \\
\hline & \multicolumn{2}{|l|}{ Fathers } & $112(42 \%)$ \\
\hline & \multicolumn{2}{|l|}{ Guardian } & $40(15 \%)$ \\
\hline \multicolumn{4}{|l|}{ Strabismus measurement } \\
\hline \multirow[t]{2}{*}{ Deviation (prism diopters, PD) } & \multicolumn{2}{|l|}{ Distance } & $10-84(29.5 \pm 11.5)$ \\
\hline & \multicolumn{2}{|l|}{ Near } & $10-84(32.5 \pm 11.0)$ \\
\hline \multirow[t]{10}{*}{ Binocular Function Stereoacuity } & \multirow[t]{3}{*}{ Distance } & Normal $(<100 \mathrm{~s}$ arc $)$, & $50(18.8 \%)$ \\
\hline & & Subnormal $(120-480 \mathrm{~s}$ arc $)$ & $44(16.5 \%)$ \\
\hline & & None $(>480 \mathrm{~s}$ arc $)$ & $172(64.7 \%)$ \\
\hline & \multirow[t]{4}{*}{ Near } & Normal $(<100 \mathrm{~s}$ arc $)$ & $78(29.3 \%)$ \\
\hline & & Subnormal (120-480 s arc) & $125(47 \%)$ \\
\hline & & None $(>800 \mathrm{~s}$ arc $)$ & $63(23.7 \%)$ \\
\hline & & Normal & $97(36.5 \%)$ \\
\hline & Distance & Abnormal (suppression or diplopia) & $169(63.5 \%)$ \\
\hline & \multirow[t]{2}{*}{ Near } & Normal & $180(67.7 \%)$ \\
\hline & & Abnormal (suppression or diplopia) & $86(32.3 \%)$ \\
\hline \multicolumn{4}{|l|}{ Control of deviation } \\
\hline \multirow[t]{4}{*}{ Newcastle home control score } & & 0 (Never) & $147(55.3 \%)$ \\
\hline & & $1(\leq 50 \%$ distance $)$ & $71(26.7 \%)$ \\
\hline & & $2(\geq 50 \%$ distance $)$ & $48(18 \%)$ \\
\hline & & 3 ( $\geq 50 \%$ distance and near) & $0(0 \%)$ \\
\hline \multirow{3}{*}{\multicolumn{2}{|c|}{ Newcastle office control at distance }} & 0 (Immediate realignment) & $63(23.7 \%)$ \\
\hline & & 1 (Realign with blink or fixation) & $81(30.5 \%)$ \\
\hline & & 2 (Remain manifest or manifest spontaneously) & $122(45.8 \%)$ \\
\hline \multirow[t]{3}{*}{ Newcastle office control at near } & & 0 (Immediate realignment) & $95(35.7 \%)$ \\
\hline & & 1 (Realign with blink or fixation) & $102(38.3 \%)$ \\
\hline & & 2 (Remain manifest or manifest spontaneously) & $69(26 \%)$ \\
\hline
\end{tabular}



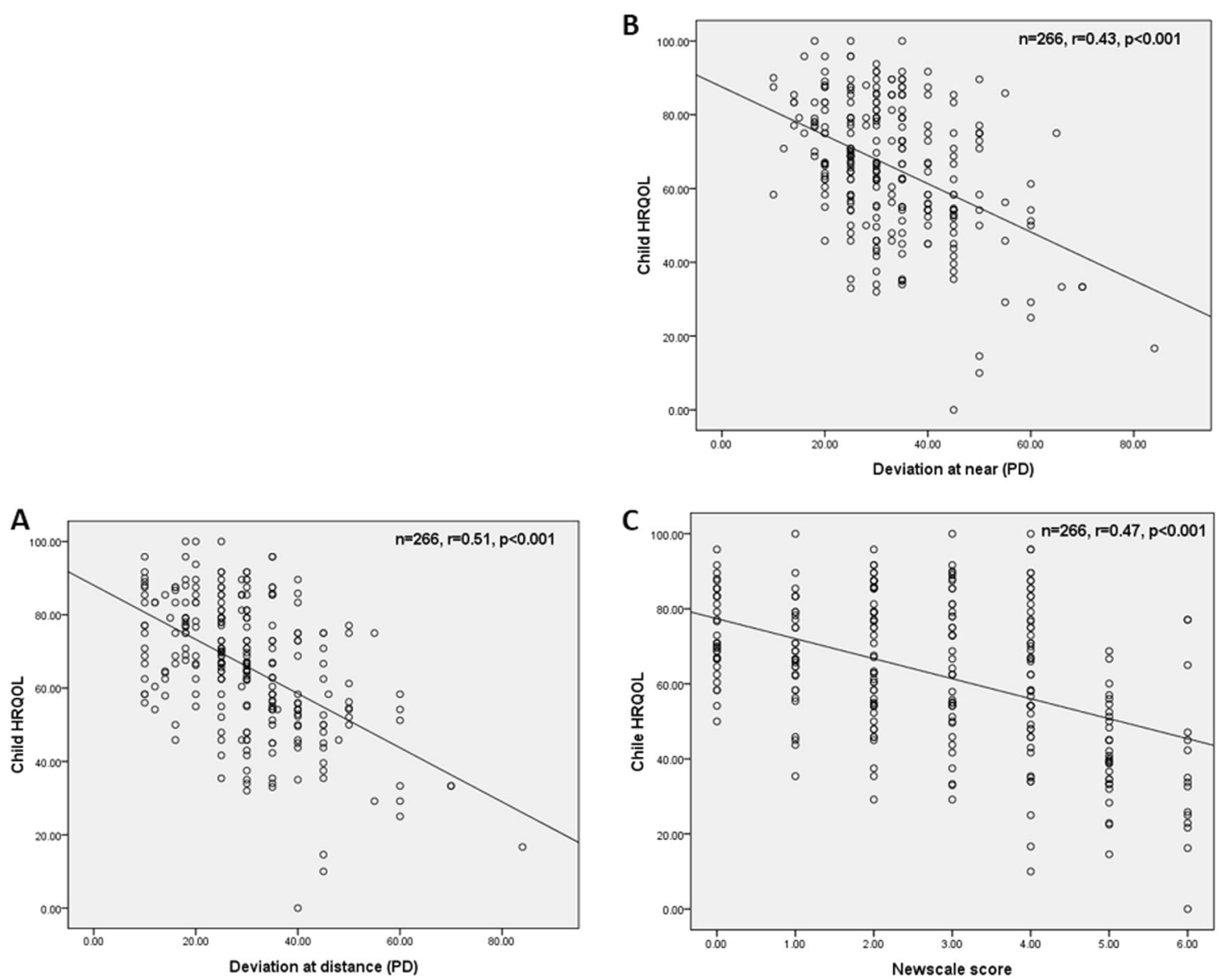

Fig. 1 a Correlation of child HRQOL and deviation at distance. b Correlation of child HRQOL and deviation at near. c Correlation of child HRQOL and Newcastle control score

A larger deviation apparently resulted in a poorer child IXTQ score (for distance, $r=0.51, p<0.001$; for near degree, $r=0.43, p<0.001)$. Moreover, poorer Newcastle control scores were associated with lower child IXTQ scores $(r=0.47, p<0.01)$ (Fig. 1c). There was a significant effect in the child IXTQ scores for the near stereo function ( $F=3.45, p=0.03$ ) but not for the distance stereo function $(F=0.96, p=0.38)$. No significant difference in the child IXTQ scores was found between the sensory fusion (for distance fusion, $t=0.62, p=0.54$; for near fusion, $t=1.87$, $p=0.063$ ).

In Fig. 2, we plotted the proxy child IXTQ scores as a function of the deviation angle at distance (Fig. 2a) and near (Fig. 2b), as well as the Newcastle control scores (Fig. 2c). Pearson correlation analysis showed a significant correlation between the proxy child IXTQ scores and these measures of intermittent exotropia severity (for distance angle, $r=0.52, p<0.001$; for near angle, $r=0.43, p<0.001$; for the Newcastle control scores, $r=0.54, p<0.001)$. Significant difference was found in the proxy child IXTQ scores between the difference stereo function (for distance stereo function, $F=5.27, p=0.006$; for near stereo function, $F=3.81, p=0.023)$, as well as between patients' sensory fusion (for distance sensory fusion, $t=2.69, p=$ 0.008 ; for near sensory fusion, $t=3.14, p=0.002$ ).

A weak but significant correlation was found between the parent IXTQ scores and deviation at distance (Fig. 3a, $r=$ $0.26, p<0.01$ ) and near (Fig. 3b, $r=0.26, p<0.01$ ), as well as with the Newcastle control scores (Fig. 3c, $r=0.14, p=$ 0.028). There was a significant difference in the Parent IXTQ scores between the children with different distance stereo function $(F=7.18, p=0.001)$ and near stereo function $(F=3.14, p=0.04)$, as well as between sensory fusion for near fusion $(t=2.22, p=0.027)$ but not for distance fusion $(t=1.53, p=0.13)$.

\section{Relationship between child and parent scores}

Pearson correlations also showed a significant correlation between the child IXTQ scores and proxy child IXTQ scores $(r=0.43, p<0.001)$, between the parent IXTQ scores and proxy child IXTQ scores $(r=0.44, p<0.001)$ and between the parent IXTQ scores and child IXTQ scores $(r=0.29, p<0.001)$. 

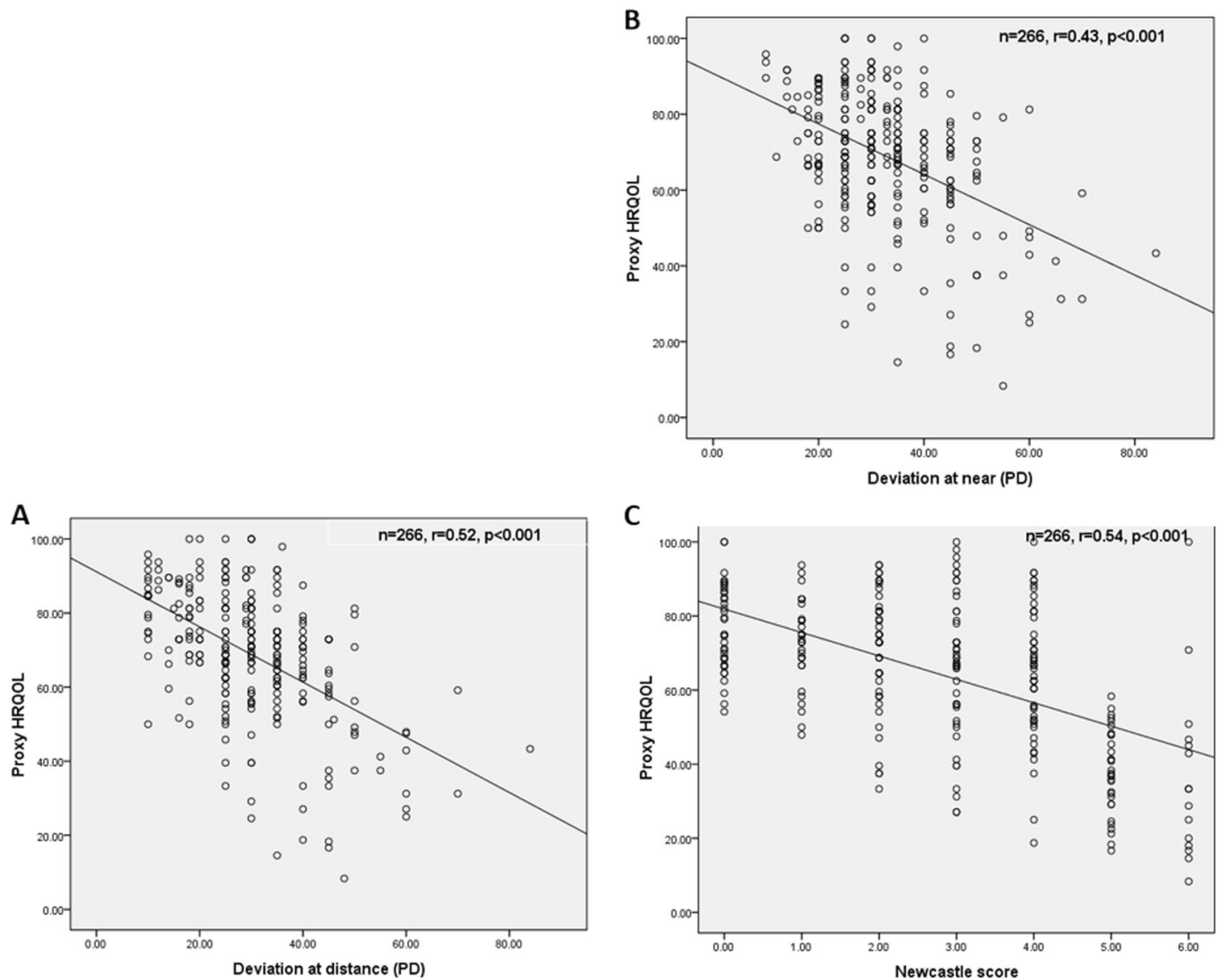

Fig. 2 a Correlation of proxy child HRQOL and deviation at distance. b Correlation of proxy child HRQOL and deviation at near. c Correlation of proxy child HRQOL and Newcastle control score

\section{Factors associated with HRQOL}

Based on the above mentioned analysis, we conducted multivariate linear regression analysis to evaluate the factors significantly associated with IXTQ scores. Deviation at distance and Newcastle home control score were significantly associated with child IXTQ scores and proxy child IXTQ scores. The stereoacuity at distance and Newcastle home control score were significantly associated with Parent IXTQ scores.

\section{Discussion}

In this study, we assessed the relationship between the severity of intermittent exotropia and HRQOL in children. Lower IXTQ scores were found in patients with the increased severity of exotropia, including a larger deviation angle, poorer control scores, and worse stereo function. In multivariate linear regression analysis, the deviation angle at distance and Newcastle control scores were significantly associated with the child and proxy child scale. The
Newcastle control score and stereo function were associated with the parent IXTQ scores. Furthermore, the Parent HRQOL score was strongly correlated with the child HRQOL and parent proxy HRQOL scores.

Our results are inconsistent with the findings of Lim et al.'s recent report in which the Child IXTQ scores were not correlated with strabismus severity, i.e., the deviation, stereo function, and Newcastle control scores [13]. Several reasons might account for this difference between the two studies. First, the correlation may not have been strong enough to be captured by the small sample size $(n=63)$ in Lim et al.'s study. Second, there is a difference in the severity of intermittent exotropia between the two studies. The deviation angle ranged from 10 to $84 \mathrm{PD}$ at distance and near in our study, while the deviation angle ranged from 14 to $60 \mathrm{PD}$ at distance and 1 to 53 PD at near in Lim et al.'s subjects. The Newcastle control scores were equally distributed from " 0 " to " 2 ", and the distribution of stereo function was relatively even in our study (i.e., $30 \%$ of subjects had normal stereo function, $45 \%$ had subnormal, and $25 \%$ had no stereo function at near). In contrast, the patients in Lim et al.'s study had more homogeneity in terms of severity, e.g., $\sim 90 \%$ of their patients 

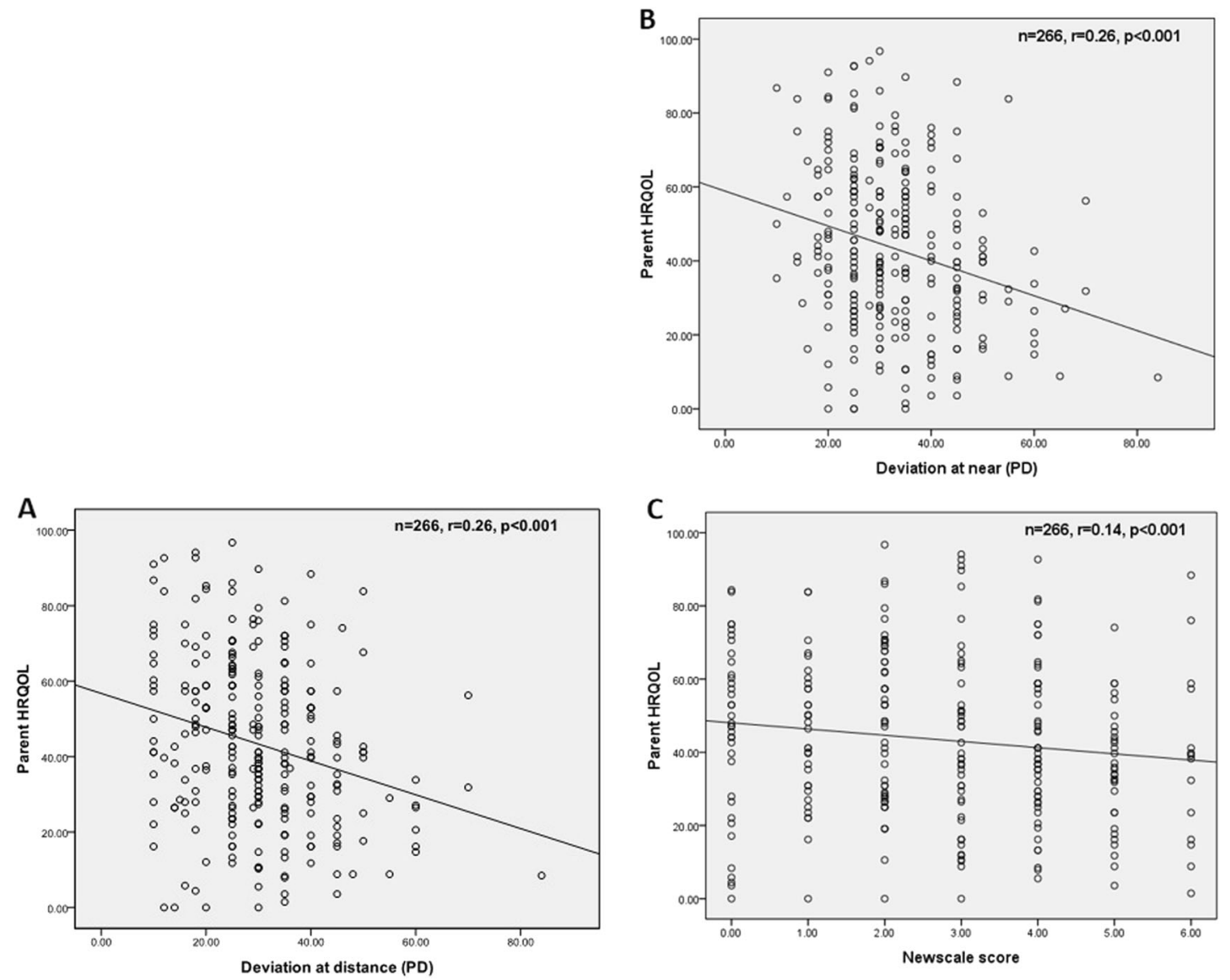

Fig. 3 a Correlation of parent HRQOL and deviation at distance. b Correlation of parent HRQOL and deviation at near. c Correlation of parent HRQOL and Newcastle control score

scored "1" or "0" on the office control score, and 71\% subjects had normal stereo function at near. In total, the different sample size, different severity of the patients in the two studies may account for these inconsistencies.

The relationships between the size and control of deviations with HRQOL are unsurprising as several previous studies also found that children with noticeable exotropia received negative social reactions from their peers [20-23] and were even subjected to negative social bias from their teachers, with a negative impact on their education and socialization [24]. Moreover, individuals with larger deviation and worse control in exotropia had more disturbances in visual performance, i.e., the size of exodeviation was associated with the binocular interaction, as well as the accommodative response during binocular vision in intermittent exotropia [25].

We found that the proxy child HRQOL and parent HRQOL were associated with the size of deviation and control score. It was also supported by previous studies, as from the parent's prospective, the "comments from others" and "worry about the surgery" were the most concerning to the parents regarding their children's intermittent exotropia
$[10,11]$.The parents may worry about the influence of the social prejudice on their child, especially if the child's squinting is more pronounced and less controlled [13].

In a recent study, no consistent correlation was found between the control, deviation angle and stereoacuity in subjects with intermittent exotropia. The study suggested that the role of exotropia control, stereoacuity and angle of deviation in the diagnosis and management of intermittent exotropia was still unclear [26]. Our study found a moderate relationship between the deviation angle, control score, stereoacuity, and HRQOL in children with intermittent exotropia, as assessed by themselves and their parents. This finding was supported by a previous study in which low proxy IXTQ and parent HRQOL scores were associated with the decision to perform surgery for children with intermittent exotropia [27]. This result implied that objective clinical measurements could reflect the subjective impact of the condition on patients and their parents. Furthermore, the subjective evaluation of the patients may be helpful for the management of intermittent exotropia.

Interestingly, in our study, patients' stereoacuity and sensory fusion played an important role in HRQOL for 
both children and their parents. According to previous studies, there might be some deficits in visual processing at the cortical level in patients with intermittent exotropia. For example, cortical activity dysfunction has been observed in several regions, including the middle occipital gyrus, fusiform gyrus, and lingual gyrus, in patients with intermittent exotropia [28]. In addition, voxel-based morphometry indicated impairment of the primary visual cortex in intermittent exotropia [29]. All these results, together with the tight link between stereopsis and HRQOL observed here, indicated that in addition to abnormal deviations, we should pay close attention to deficits in visual cortical processing. In fact, a recent study in surgically corrected intermittent exotropia found that the sensory eye balance in binocular phase combination remains abnormal [30].

Our study had some limitations. Stereoacuity, deviation, and control are variable in intermittent exotropia $[6,8]$. Only one evaluation of these measurements, including exodeviation control, was obtained at each distance in the study. The evaluation could be affected by the bias from several sources. For example, the Newcastle control score used in the study was assessed by the same investigator who also evaluated the HRQOL. Some items included in the Newcastle score were evaluated by the parents of the patients. Other factors, such as the patients who may had poorer binocular function as compared with previous study [5], and the duration of the patients with the intermittent exotropia may affect our measurement as well. Recent studies have suggested using the Office Control Score for IXT, which involves control evaluation by the physician only in the office [31]. Previous studies have suggested that performing three nonconsecutive measurements of exotropia control and obtaining the mean of the three separate measures to provide a more representative assessment of an individual's IXT control [7, 32]. Furthermore, a revised Newcastle control score which changed score 7 to 9 by adding score 3 for control evaluation in office [33], it would be helpful for assessed the patients present manifest exotropia spontaneously, especially for distance. The angle of deviation was obtained by measuring the maximum deviation through breaking fusion using PACT, which is different from the manifest deviation through the simultaneous prism and cover test. Thus, the results of the correlation may be different between the deviation and HRQOL. Finally, our results are not generalizable to all subjects with intermittent exotropia because the subjects who participated in this study were willing to seek treatment in the hospital, which biases the quality of life evaluation. Subjects in our study had worse binocular function, i.e., one quarter of children assessed as no stereo function at near, which was different with previous study reports, as Buck et al. reported almost of their subjects aged $\geq 4$ years had near stereo function. It may be due to the deviation last time and the methods of measurement for stereo function, as the Randot stereo test was used in our study while the Frisby was used in the Buck's study [5]. Enrolling patients in an epidemiological study may minimize the selection bias.

In conclusion, the HRQOL of both children and their parents correlated with the severity of intermittent exotropia. The larger deviation angle, poor control, and worse stereo function may predict a more negative impact on children and their parents' lives.

\section{Summary}

\section{What was known before}

- Children with intermittent exotropia would experience a negative impact on their health-related quality of life (HRQOL). Few studies have evaluated the correlation between the clinical severity and their HRQOL. Whether HRQOL is correlated with the clinical severity of this condition remains uncertain. Moreover, whether the HRQOL of children and their parents could be predicted based on the clinical evaluation of the intermittent exotropia remains unknown.

\section{What this study adds}

- HRQOL of both children and their parents correlated with the severity of intermittent exotropia. The larger deviation angle, poor control, and worse stereo function may predict a more negative impact on children and their parents' lives.

Acknowledgements This work is supported by Wenzhou Municipal Science and Technology Bureau (subject numbers: Y20160150; Y20170782) and Huzhou Municipal Science and Technology Bureau (subject number: 2017GYB13).

\section{Compliance with ethical standards}

Conflict of interest The authors declare that they have no conflict of interest.

Publisher's note: Springer Nature remains neutral with regard to jurisdictional claims in published maps and institutional affiliations.

\section{References}

1. Govindan M, Mohney BG, Diehl NN, Burke JP. Incidence and types of childhood exotropia, apopulation-based study. Ophthalmology. 2005;112:104-8. 
2. Pan CW, Zhu H, Yu JJ, Ding H, Bai J, Chen J, et al. Epidemiology of intermittent exotropia in preschool children in China. Optom Vis Sci. 2016;93:57-62.

3. Fu J, Li SM, Liu LR, Li JL, Li SY, Zhu BD, Li H. et al. Prevalence of amblyopia and strabismus in a population of 7th-grade junior high school students in Central China:the Anyang Childhood Eye Study (ACES). Ophthalmic Epidemiol. 2014;21:197-203.

4. Chia A, Roy L, Seenyen L. Comitant horizontal strabismus: an Asian perspective. Br J Ophthalmol. 2007;91:1337-40.

5. Buck D, Powell C, Cumberland P, Davis H, Dawson E, Rahi J. et al. Present features early manag child inter exotropia UK: inception cohort. Br J Ophthalmol. 2009;93:1620-4.

6. Hatt SR, Mohney BG, Leske DA, Holmes JM. Variability of control in intermittent exotropia. Ophthalmology. 2008;115:371-6.

7. Hatt SR, Mohney BG, Leske DA, Holmes JM. Quantifying variability in the measurement of control in intermittent exotropia. J AAPOS. 2015;19:33-37.

8. Hatt SR, Mohney BG, Leske DA, Holmes JM. Variability of angle of deviation measurements in children with intermittent exotropia. J AAPOS. 2012;16:120-4.

9. Hatt SR, Leske DA, Holmes JM. Awareness of exodeviation in children with intermittent exotropia. Strabismus. 2009;17:101-6.

10. Yamada T, Hatt SR, Leske DA, Holmes JM. Health-related quality of life in parents of children with intermittent exotropia. $\mathrm{J}$ AAPOS. 2011;15:135-9.

11. Hatt SR, Leske DA, Adams WE, Kirgis PA, Bradley EA, Holmes JM. Quality of life in intermittent exotropia: child and parent concerns. Arch Ophthalmol. 2008;126:1525-9.

12. Yamada T, Hatt SR, Leske DA, Holmes JM. Specific healthrelated quality of life concerns in children with intermittent exotropia. Strabismus. 2012;20:145-51.

13. Lim SB, Wong WL, Ho RC, Wong IB. Childhood intermittent exotropia from a different angle: does severity affect quality of life? Br J Ophthalmol. 2015;99:1405-11.

14. Hatt SR, Leske DA, Yamada T, Bradley EA, Cole SR, Holmes JM. Development and initial validation of quality-of-life questionnaires for intermittent exotropia. Ophthalmology. 2010;117:163-8.

15. Hulley SB, Cummings SR, Browner WS, Grady D, Newman TB. Designing clinical research: an epidemiologic approach. 4th ed. Philadelphia, PA: Lippincott Williams \& Wilkins; 2013. Page 79.

16. Guo DD, Wu JF, Hu YY, Sun W, Lv TL, Jiang WJ, et al. Stereoacuity and related factors: the Shandong Children Eye Study. PLoS ONE. 2016;11:e0157829 https://doi.org/10.1371/ journal.pone.0157829

17. Haggerty H, Richardson S, Hrisos S, Strong NP, Clarke MP. The Newcastle Control Score: a new method of grading the severity of intermittent distance exotropia. Br J Ophthalmol. 2004;88:233-5.

18. Buck D, Hatt SR, Haggerty $H$, Hrisos S, Strong NP, Steen NI, et al. The use of the Newcastle Control Score in the management of intermittent exotropia. Br J Ophthalmol. 2007; 91:215-8.

19. Zhu H, Xu S, Leng Z, Fu Z, Xiao Y, Liu H. Utilization of quality of life assessment questionnaire for intermittent exotropia in China. Chin J Ophthalmol. 2016;52:596-603.

20. Lukman H, Kiat JE, Ganesan A, Chua WL, Khor KL, Choong YF. Strabismus-related prejudice in 5-6-year-old children. $\mathrm{Br} \mathbf{J}$ Ophthalmol. 2010;94:134-51.

21. Lukman H, Kiat JE, Ganesan A, Chua WL, Khor KL, Choong YF. Negative social reaction to strabismus in schoolchildren ages 8-12 years. J AAPOS. 2011;15:238-40.

22. Paysse EA, Steele EA, McCreery KM, Wilhelmus KR, Coats DK. The emergence of negative attitudes toward strabismus. J AAPOS. 2001;5:361-6.

23. Mojon-Azzi SM, Kunz A, Mojon DS. Strabismus and discrimination in children: are children with strabismus invited to fewer birthday parties? Br J Ophthalmol. 2011;95:473-6.

24. Uretmen O, Egrilmez S, Kose S, Pamukçu K, Akkin C, Palamar M. Negative social bias against children with strabismus. Acta Ophthalmol Scand. 2003;81:138-42.

25. Ahen SJ, Yang HK, Hwang JM. Binocular visual acuity in intermittent exotropia: role of accommodative convergence. Am J Ophthalmol. 2012;154:981-6.

26. Superstein R, Dean TW, Holmes JM, et al. Relationship among clinical factors in childhood intermittent exotropia. J AAPOS. 2017;21:268-73.

27. Hatt SR, Leske DA, Liebermann L, Mohney BG, Brodsky, Yamada T, et al. Associations between health-related quality of life and the decision to perform surgery for childhood intermittent exotropia. Ophthalmology. 2014;121:883-8.

28. Li Q, Bai J, Zhang J, Gong Q, Liu L. Assessment of cortical dysfunction in patients with intermittent exotropia: an fMRI study. PLoS One. 2016;11:e0160806

29. Chan ST, Tang KW, Lam KC, Chan LK, Mendola JD, Kwong KK. Neuroanatomy of adult strabismus: a voxel based morphometric analysis of magnetic resonance structural scans. Neuro Image. 2004;22:986-94.

30. Feng L, Zhou J, Chen L, Hess RF. Sensory eye balance in surgically corrected intermittent exotropes with normal stereopsis. Sci Rep. 2015;5:13075.

31. Mohney BG, Holmes JM. An office-based scale for assessing control in intermittent exotropia. Strabismus. 2006;14:147-50.

32. Hatt SR, Liebermann L, Leske DA, Mohney BG, Holmes JM. Improved assessment of control in intermittent exotropia using multiple measures. Am J Ophthalmol. 2011;152:872-6.

33. Buck D, Clarke MP, Haggerty H, Hrisos S, Powell C, Sloper J, et al. Grading the severity of intermittent distance exotropia: the revised Newcastle Control Score. Br J Ophthalmol. 2008; 92:577. 